\title{
Numerical Study on Pressure Fluctuations Reduction in Centrifugal Pumps: Influence of Radial Gap and Splitter Blades
}

\author{
Moisés Solis, Farid Bakir, Sofiane Khelladi, and Ricardo Noguera \\ DynFluid Laboratory, Arts et Metiers ParisTech, 151, Boulevard de l'Hôpital, 75013 Paris, France \\ Correspondence should be addressed to Moisés Solis, moises.solis@yahoo.fr
}

Received 29 May 2011; Accepted 29 June 2011

Academic Editors: F. M. Gerner and S. C. M. Yu

Copyright () 2011 Moisés Solis et al. This is an open access article distributed under the Creative Commons Attribution License, which permits unrestricted use, distribution, and reproduction in any medium, provided the original work is properly cited.

\begin{abstract}
Numerical study was carried out in four centrifugal pumps to analyze the effects of adding splitters and incrementing the radial gap in order to decrease the pressure fluctuations at the blade passage frequency. It is well known that in this kind of pump, the interactions between the flow leaving the impeller and entering the volute generate fluctuating pressures which propagate toward the inlet and the discharge of the pump. These pulsations are mainly associated to the blade passage frequency presenting high amplitudes near the volute tongue and at impeller discharge. Four configurations were analyzed using numerical simulations; the first is the reference pump which will be optimized and the others are the optimized ones. The numerical modelling was performed at the same flow rate having to vary in some cases the rotational speed to reach the operating point. During the calculations, the pressure signals were recorded by virtual sensors placed at several locations of the pump then they were analyzed and processed by means of the Fast Fourier Transform. The pressure fluctuations were plotted and compared between the four configurations, showing clearly the fact of varying the blades number and the radial gap influence significantly on their reduction.
\end{abstract}

\section{Introduction}

Nowadays, the application of centrifugal pumps has been extended in several domains of industry as well as the research works to improve and to understand the flow inside this kind of turbomachines. Designing an efficient volute type centrifugal pump responding to acoustic and hydraulic specifications is very complex despite technological advances in this domain. The main obstacle for pump designers is the large quantity of geometrical parameters necessary to take into account in the optimization process. It is known that the pump during its operation is a source of instabilities and noise due mainly to the operating conditions and the strong interactions between the impeller blades and the volute tongue. Many research works concerning the study of the physical mechanisms and the proposal of classical solutions based on intuition and parametric studies to control or reduce specially the tonal noise have been made. Although, not only the interaction blade/tongue is the main source that contributes to pressure fluctuations, the presence of instabilities other than blade passage is also indirectly involved in the generation of noise. This aspect was reported by Choi et al. [1] who performed an experimental investigation about the process of noise generation related to the flow field instabilities in a pump rotor. He could show that the jet-wake flow pattern is a source of noise generation near to blades trailing edge, and it induces a periodic pressure fluctuations on the blade surfaces. According to the literature, Majidi [2] investigated by numerical simulations the unsteady periodic behavior of the flow in a classic pump, noticing that the flow is characterized with pressure fluctuations due to the interaction between the impeller and the volute casing. Spence and Amaral-Teixeira [3] worked in the investigation of the pressure pulsations in a centrifugal pump, measuring in various positions the pulsations levels and finding strong amplitudes at the impeller outlet. Moreover, the asymmetric shape of the volute and tongue is a source of circumferential distortion of pressure distribution according to Asuaje et al. [4], who did experiments that showed the influence of volute tongue causing an unsymmetrical flow distribution and nonuniform pressure distribution at impeller periphery. Many works in experimental and numerical investigation have been done helping the understanding of the geometrical factors influence and operating specifications and their 


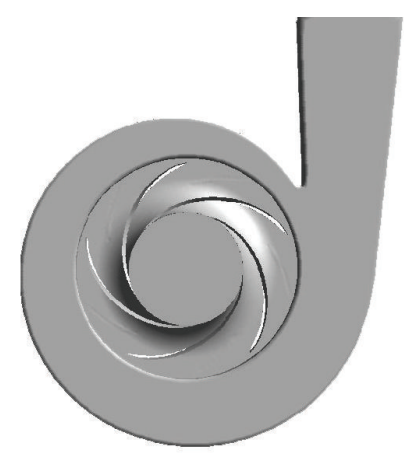

(a) $\mathrm{R} 1 \mathrm{~A}+\mathrm{V} 1$

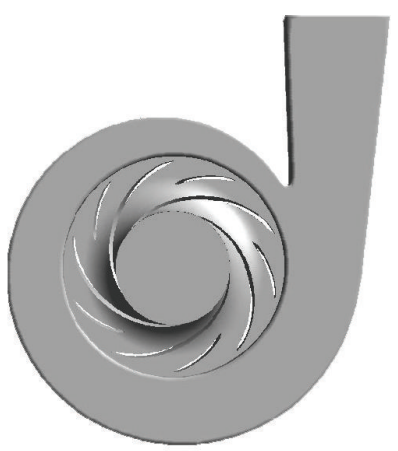

(c) R1AS+V1

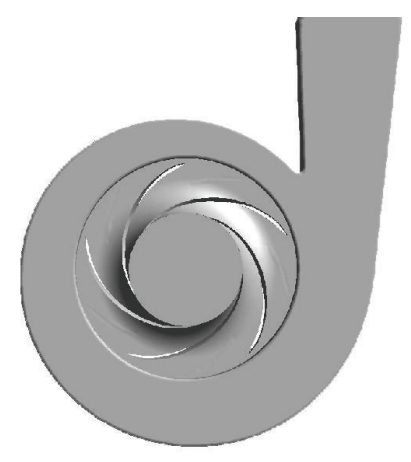

(b) $\mathrm{R} 1 \mathrm{~A}+\mathrm{V} 2$

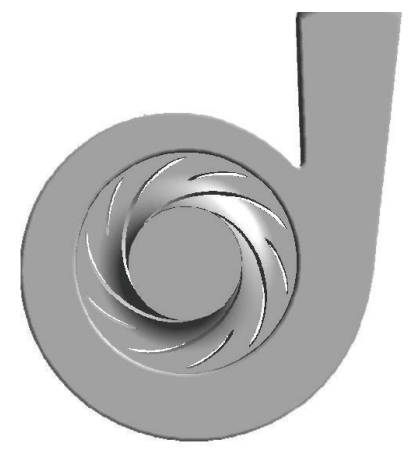

(d) R1AS+V2
Figure 1: Pump configurations.

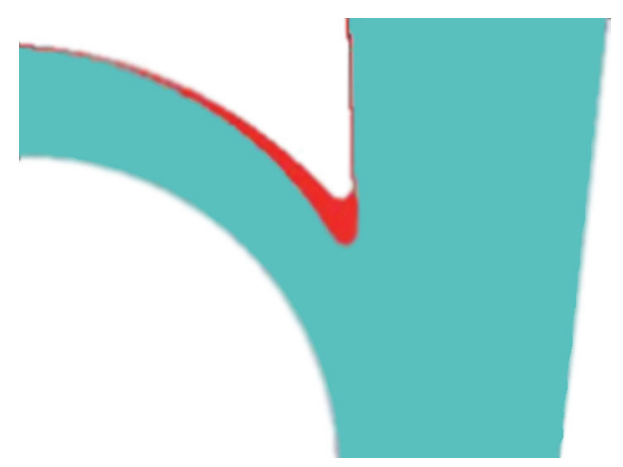

Figure 2: Detail of radial gap increment.

relation with the pressure fluctuating behavior aiming to decrease the noise levels inside the pump. One of such factors is the increasing of the number of blades which has a positive impact on the acoustic behavior but decreases the hydraulic pump efficiency, [5]. Also Kergourlay et al. [5], who experimented for various flow rates the presence of splitter blades, shows that this late solution has a positive effect on the pressure fluctuations reduction at the discharge pump. Also he found experimentally and numerically that the use of intermediary shorten blades allows a better circumferential pressure distribution. Otherwise, Miyamoto et al. [6], who worked on experimental measurements in an unshrouded and a shrouded impeller with splitter blades, showed that with splitters the blade loadings tend to

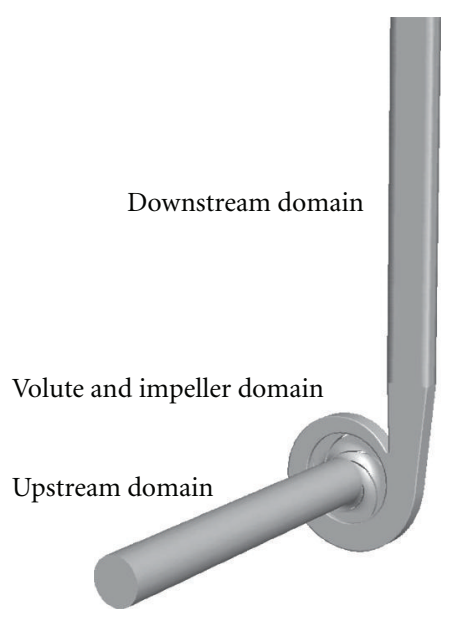

FIGURE 3: 3D Model of numerical domain for R1A+V1.

become smaller, and the absolute circumferential velocities and total pressures become considerably larger than those impellers without splitter blades, thus noticing the influence of the splitter blades on static pressure. Another important consideration about pressure fluctuations is the operating point, Parrondo-Gayo et al. [7] pointed out through experimental measurements in the volute of a centrifugal pump the influence of operating point on pressure fluctuations at the blade passage frequency, finding smaller values as it approaches to design flow rate. It is known that the excitations generated by the blade passage near the volute tongue can be reduced to a certain level by means of increasing radial gap. In previous research works Dong et al. [8-10] studied the radial gap influence on the pressure fluctuations reduction, in order to achieve these geometrical changes; they modified the volute tongue position keeping the same impeller geometry. González et al. [11] investigated numerically and experimentally the influence of radial gaps. They used two impellers with different diameters which were tested in the same volute. The results determined that there is not a strong effect on the pressure fluctuations at any frequency and, in particular, at the blade passage frequency. Recently, Barrio et al. [12] worked on the fluid dynamic pulsations at the blade passage frequency and their relation with the impeller cutback, investigating the effect on the pressure fluctuations of progressive augmentation of tongue radial gap by means of trimming the impeller.

The present paper focuses on the numerical study of pressure fluctuations reduction by means of geometric changes of the impeller geometry and the increment of radial gap. Comparisons of pressure fluctuations amplitudes were made between the four configurations in various forms. The numerical procedure used was determined after investigations about the boundary conditions influence assuming a condition of fully-developed flow at outlet domain. To achieve the numerical purposes, four configurations were modeled that correspond to the initial not optimized geometry and the three improved configurations to reduce pressure fluctuations amplitudes. The numerical simulations were based on the Unsteady Reynold Averaged Navier Stokes 


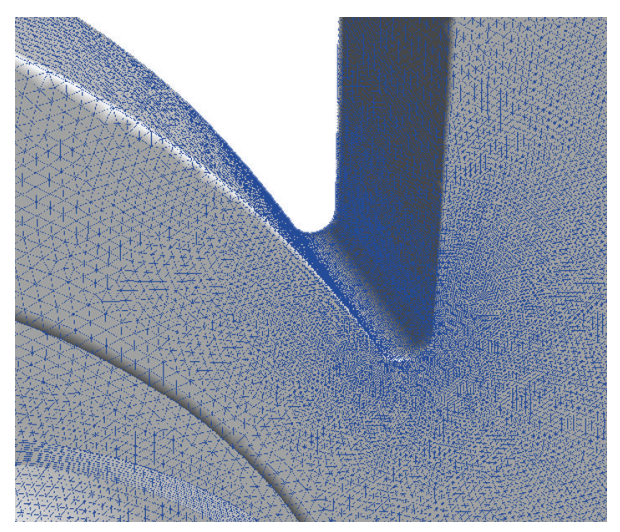

(a) Volute tongue

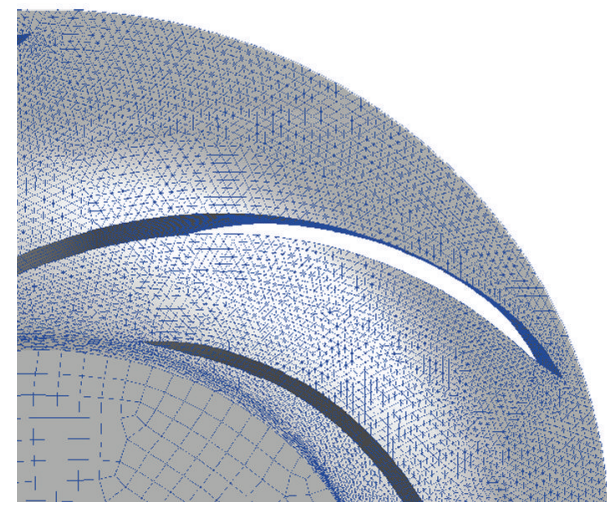

(b) Trailing edge

FIGURE 4: Sketch of unstructured mesh for geometry R1A+V1.

approach and the Fluent code [13] was used to solve the fluid governing equations. For all configurations, the tests have been carried out for the same flow rate. An adjustment in rotational speed was considered for some configurations in order to work at the same operating point.

\section{Pumps Presentation and Operating Specifications}

The reference geometry to be optimized is called R1A+V1, it is a single-stage volute type centrifugal pump which rotates at $2930 \mathrm{rpm}$. The impeller R1A is semiopen and has five backward curved vanes varying its geometric profile from the shroud to the hub. The volute V1 is a classic one having rectangular sections with symmetric shape, and the radial gap considered is equal to $22 \%$ of impeller radius. In order to study the influence of geometry impeller and volute tongue position, three other configurations were obtained from the main geometry. The first modified configuration is $\mathrm{R} 1 \mathrm{~A}+\mathrm{V} 2$, which was built by increasing the radial gap to $25 \%$ of impeller radius obtaining by this way a new volute V2 (see Figure 2). Then, R1AS+V1 was created by adding five intermediary shorten blades between the main blades of R1A in the middle of the outlet passage to form the new impeller

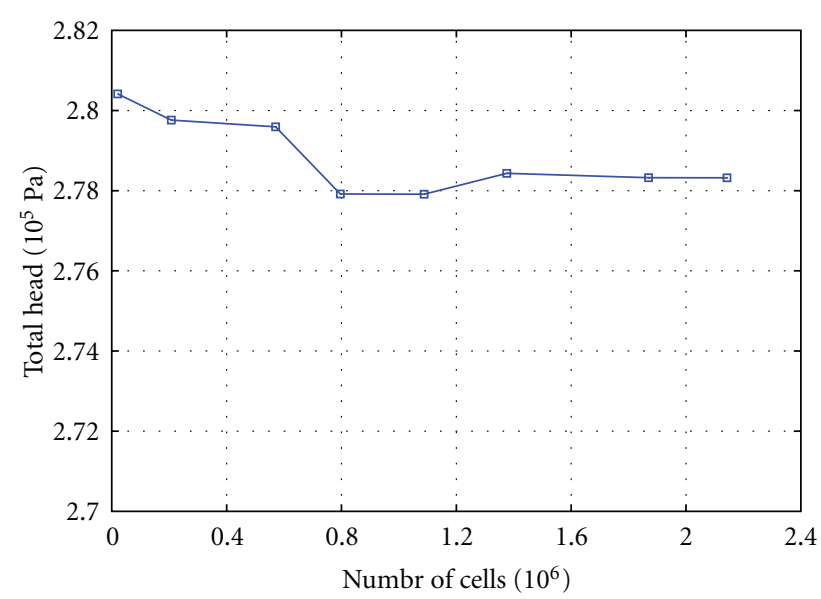

FIGURE 5: Influence of mesh on the total head for the original pump.

TABLE 1: Main characteristics of initial pump to optimize.

\begin{tabular}{lc}
\hline \multicolumn{2}{c}{ Impeller and volute } \\
\hline Total blades number, $z$ & 5 \\
Impeller outlet passage width, $b_{2}$ & $13.44 \mathrm{~mm}$ \\
Impeller inlet diameter, $d_{1}$ & $83.05 \mathrm{~mm}$ \\
Impeller outlet diameter, $d_{2}$ & $160 \mathrm{~mm}$ \\
Volute width at base circle, $b_{3}$ & $24 \mathrm{~mm}$ \\
Volute base circle diameter, $d_{3}$ & $195.2 \mathrm{~mm}$ \\
\hline \multicolumn{2}{c}{ Operation point } \\
\hline Flow rate, $Q_{N}$ & $60 \mathrm{~m}^{3} / \mathrm{h}$ \\
Total head, $H_{N}$ & $28.4 \mathrm{~m}$ \\
Rotational speed, $N$ & $2930 \mathrm{rpm}$ \\
\hline
\end{tabular}

R1AS, keeping the same discharge blade angle and the same volute V1. The third optimized configuration R1AS+V2 is a combination of the impeller with splitter blades and the volute V2 defined previously. The main dimensions and characteristics of the reference pump and the operation point are summarized in Table 1, and the different configurations can be seen in Figure 1.

\section{Numerical Modeling}

To predict pressure fluctuations generated by the interaction between the rotating impeller blades and the stationary pump casing and to model the internal flow, numerical simulations were carried out using a computational fluid dynamic code which is based on the finite volume numerical method. The governing fluids equations such as continuity, momentum, and turbulence equations were solved in the steady state calculations using the moving reference frame, while for the unsteady calculations the sliding mesh technique was applied, allowing to simulate the threedimensional flow inside the pump and the interactions between the impeller and the volute. The turbulence effects were simulated by using the $k$ - $\omega$ SST turbulence model and the boundary conditions concern a uniform normal velocity 


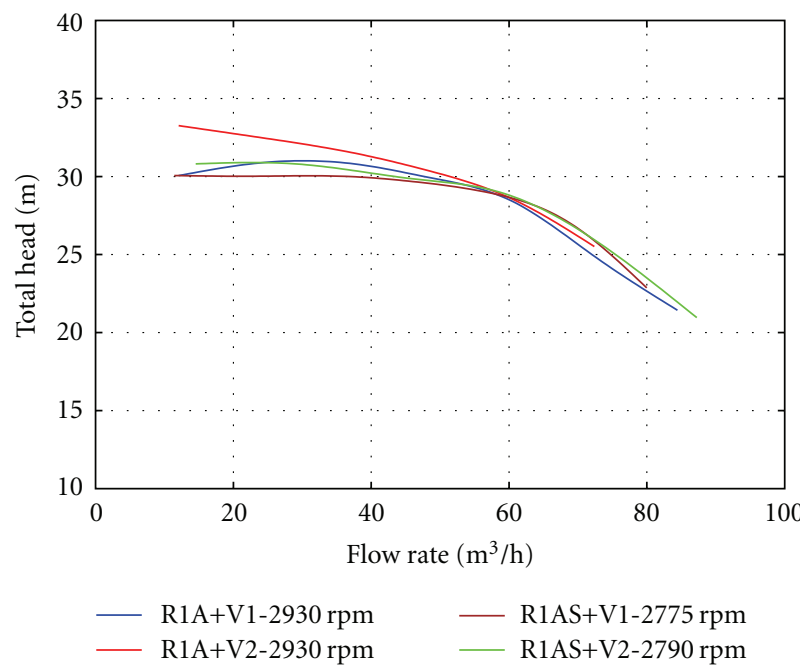

(a) $\mathrm{Q}-\mathrm{H}$

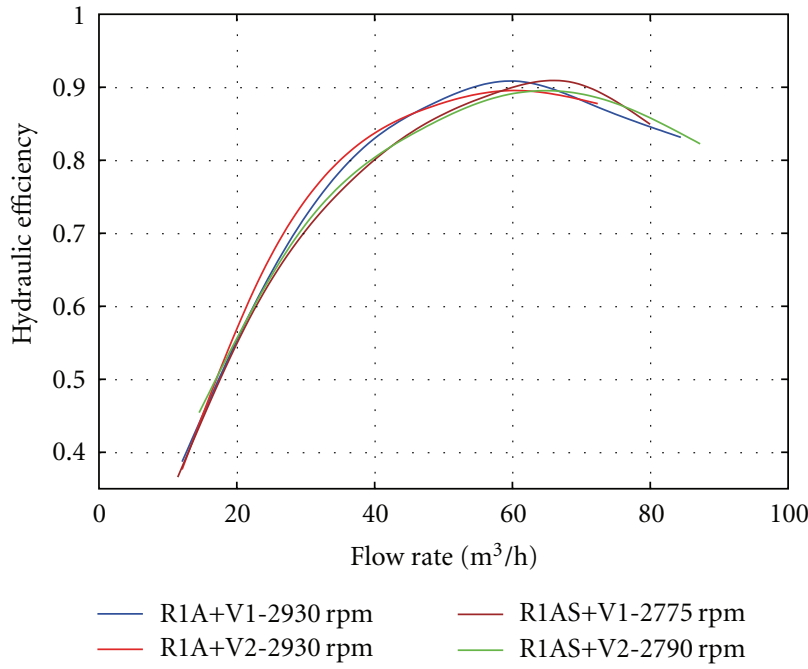

(b) $Q-\eta$

FIGURE 6: Comparison between the four geometries of characteristics curves.

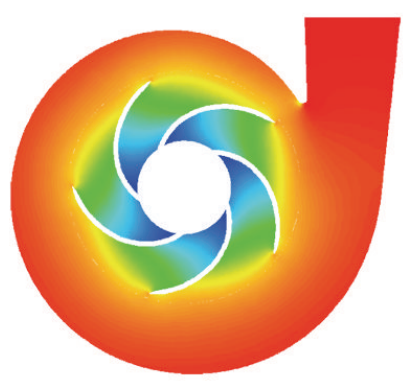

(a) $\mathrm{R} 1 \mathrm{~A}+\mathrm{V} 1$

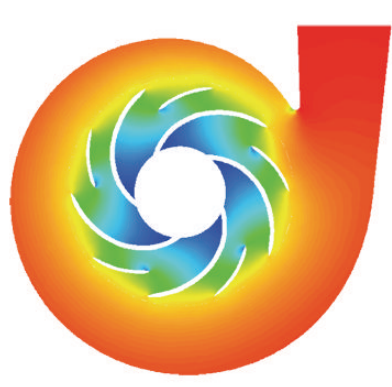

(c) R1AS+V1

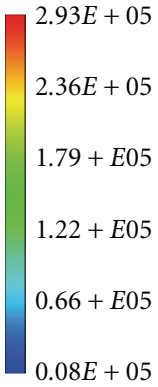

$2.93 E+05$
$2.36 E+05$
$1.79+E 05$
$1.22+E 05$
$0.66+E 05$
$0.08 E+05$

Figure 7: Pressure fields $(\mathrm{Pa})$ at blade passage frequency, at midspan of impeller outlet width.

at the inlet and outflow at the outlet of the computational domain. The capabilities of the numerical model are mainly focused on preventing any impact on the numerical results specially in the discharge channel, so an investigation of the influence of boundary condition was made testing various configurations (see [14] for more details). The results concluded that the fact of assuming the flow close to a fully developed condition at outlet provides better results concerning the pressure amplitudes levels while a pressure

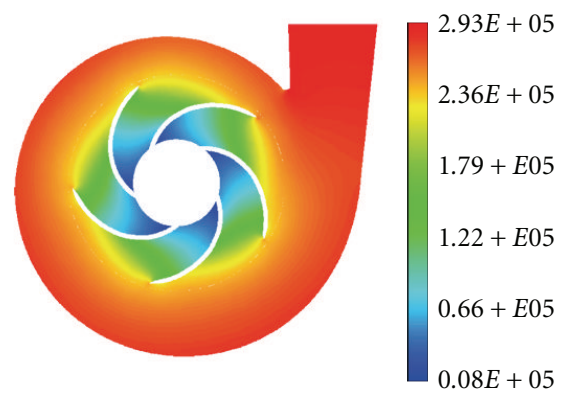

(b) $\mathrm{R} 1 \mathrm{~A}+\mathrm{V} 2$

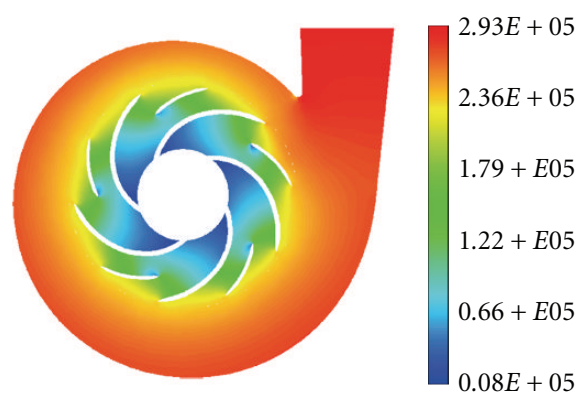

(d) R1AS+V2 outlet imposed at the outlet has an important influence on the pressure fluctuations. A second order implicit scheme was used for the time dependent terms. The pressurevelocity coupling is achieved using the SIMPLE algorithm. On the other hand; a second-order upwind discretization is used for convection terms and central difference schemes for diffusion terms. To ensure numerical stability and to minimize boundary conditions effects, the inlet and outlet ducts were extended as we can see in Figure 3. For each 


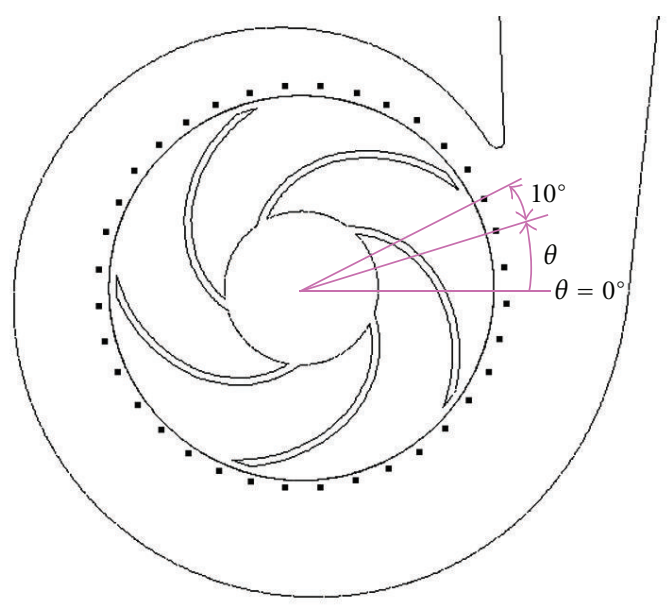

(a) Frontal view

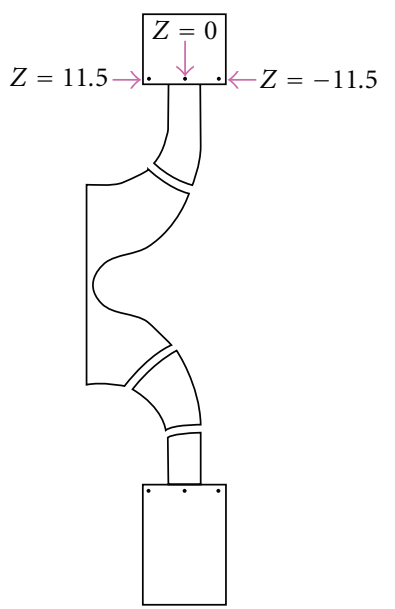

(b) Meridional view

FIgURE 8: Location of measurement points for configurations $\mathrm{R} 1 \mathrm{~A}+\mathrm{V} 1$ and $\mathrm{R} 1 \mathrm{AS}+\mathrm{V} 1$.

configuration a computational domain was built separating it in four zones in order to model the $3 \mathrm{D}$ flow. So it was obtained the upstream, impeller, volute, and downstream zones. For all simulations, a hybrid mesh was applied for each configuration. Structured hexahedral cells were used in the inlet and outlet domain, and unstructured tetrahedral cells were used for the impeller and volute casing. A special refinement is applied in the tongue region and less refined around the blades (details in Figure 4). The comparison of results between the four geometries consider approximately the same quantity of elements trying to get the same distribution in each subdomain. The front and rear axial gap between the impeller and the volute casing sidewalls were not modeled in the calculations. The time step for the unsteady calculations has been set to $5 \cdot 10^{-5} \mathrm{~s}$ allowing to have a good resolution in time. The unsteady simulation is initiliazed from the solution of a steady calculation; by this way the calculations are carried out until the convergence is reached. The static pressure signal recorded by each outlet virtual sensor was considered to determine if the calcul achieves the convergence. Due to the geometrical complexity of

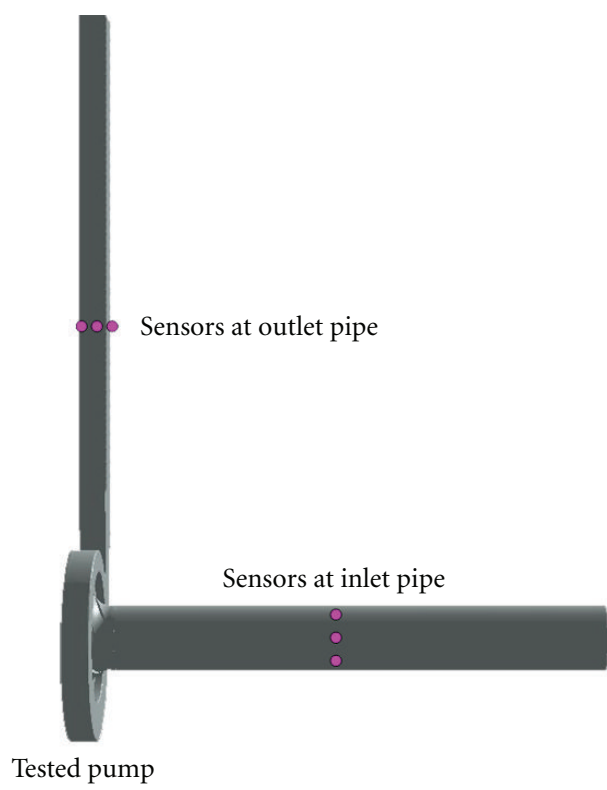

FIGURE 9: Location of measurement points at inlet and at outlet pipes for the four pump configurations.

the pumps, the size of the mesh that will be used is not the most adequate to investigate local boundary layer variables, but the global ones will be calculated using a wall function treatment based on the logarithmic law.

The number of elements used during the calculations was chosen after a mesh independence analysis on the total head (see Figure 5); this study was carried out using a steady state calculations. So, approximately 1.4 million cells were used for the bulk of the simulations which ensure the independency of the numerical results (it was observed a difference of about 0.04\% concerning the total head between the largest mesh, and the considered mesh). Table 2 summarizes the grid size employed for each modeled subdomain in the numerical tests. On the other hand, for all configurations a specific number of revolutions are necessary in the simulations to achieve a stable periodic solution, so the evolution in time of static pressure was recorded and then they were analyzed in the frequency domain using the Fast Fourier Transform. With the steady calculations, it was possible to get information on total head and hydraulic efficiency in order to compare, for the operating point, how the geometrical modifications had affected the hydraulic behavior as well as the global performances.

\section{Numerical Results}

4.1. Characteristic Curves of Tested Pumps. Prior to the unsteady calculations, it is necessary to construct the performance curves for the four configurations at $2930 \mathrm{rpm}$. Then, similarity laws will be used to determine the appropriate rotational speed (only for geometries that do not meet the operating point for example R1AS+V1 and R1AS+V2) in order to respect the specifications. In the case of $\mathrm{R} 1 \mathrm{~A}+\mathrm{V} 2$, the modification made to the volute did not have an important 


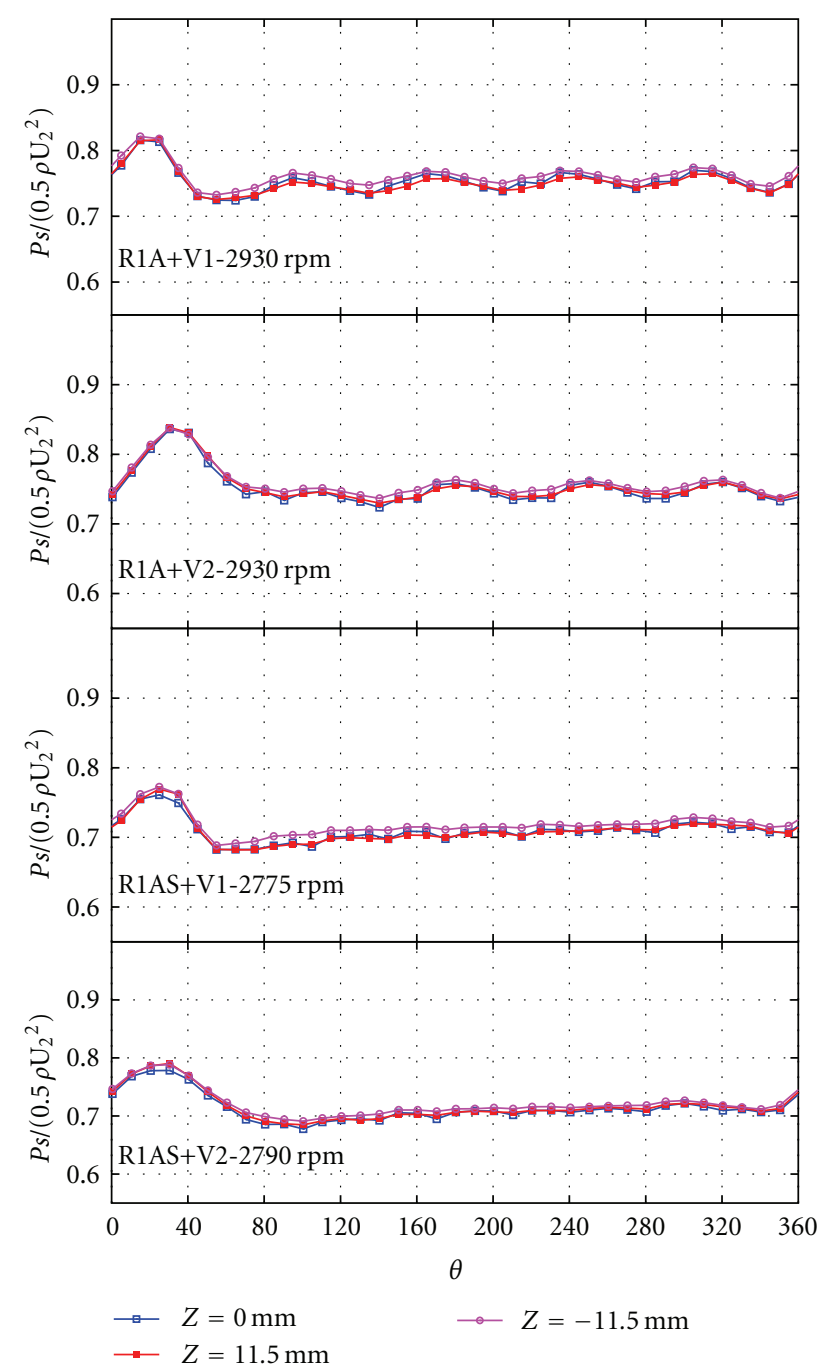

Figure 10: Circumferential distribution of normalized static pressure for the four pump configurations close to impeller exit at three axial planes, at $t=0.5$. Vertical dashed line indicates position of tongue.

TABLE 2: Total cells number for pump configuration R1A+V1.

\begin{tabular}{lc}
\hline Zone & Cells number \\
\hline Suction pipe & 138596 \\
Impeller & 629920 \\
Volute casing & 609126 \\
Discharge pipe & 40470 \\
\hline Total & 1418112 \\
\hline
\end{tabular}

effect. So, the numerical performances curves for the tested pumps were obtained and compared to the nominal point of the reference pump R1A+V1. In Figure 6, the global stationary pump performances for each configuration are presented. It can be seen that the nominal point for the original configuration is found at $Q_{N}=60 \mathrm{~m}^{3} / \mathrm{h}$ and $H_{N}=$ $28.6 \mathrm{~m}$; obviously the geometric changes had moved it in the other configurations, in doing that they work under their

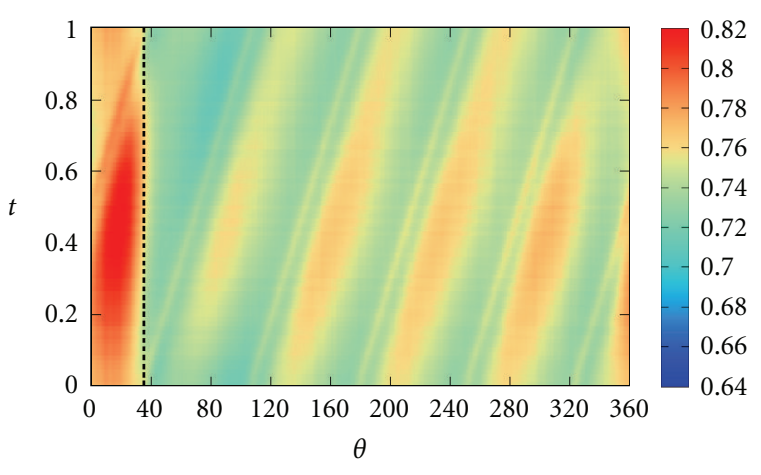

(a) $\mathrm{R} 1 \mathrm{~A}+\mathrm{V} 1$

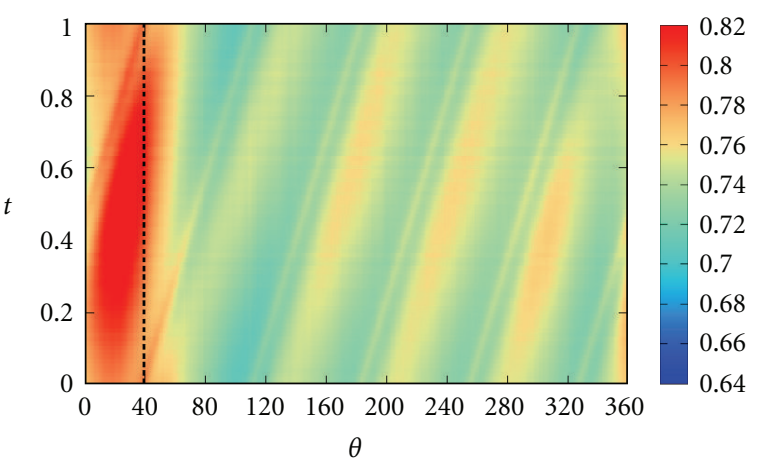

(b) $\mathrm{R} 1 \mathrm{~A}+\mathrm{V} 2$

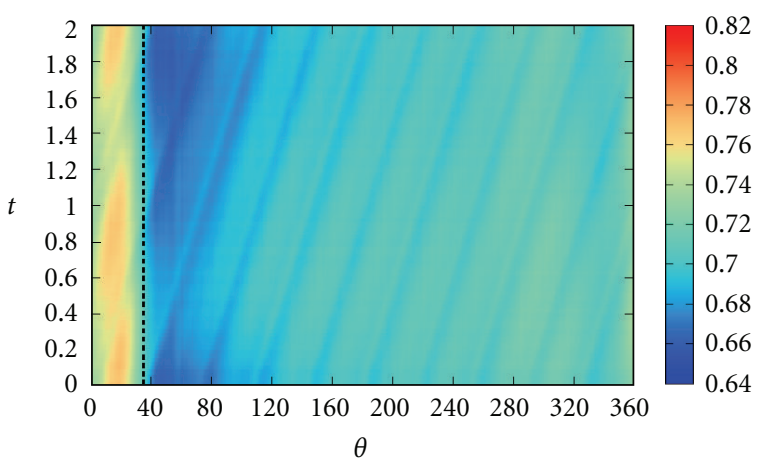

(c) R1AS+V1

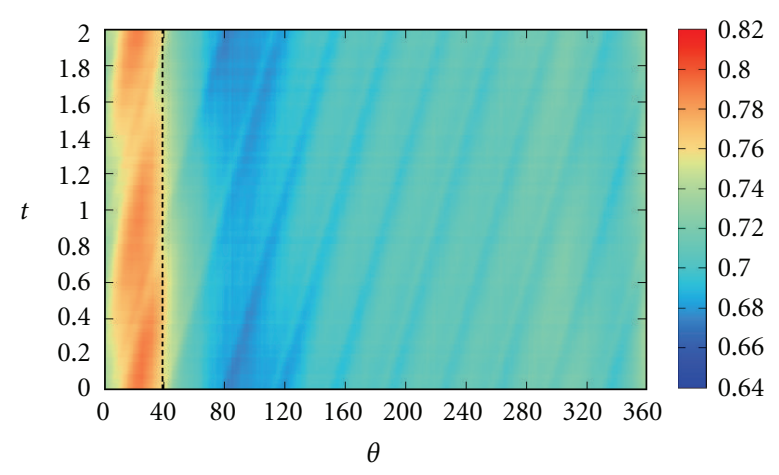

(d) R1AS+V2

FIGURE 11: Time-Spatial behavior of pressure fluctuations as function of angular position at impeller volute interface for the four pump configurations, at midspan impeller outlet. 


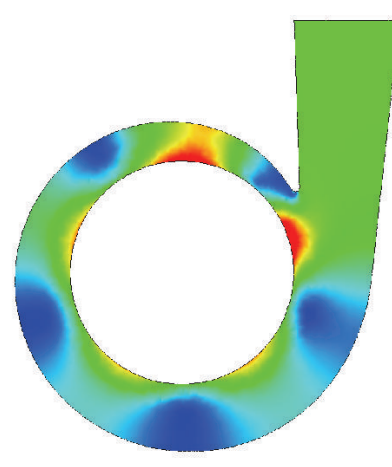

(a) $\mathrm{R} 1 \mathrm{~A}+\mathrm{V} 1$

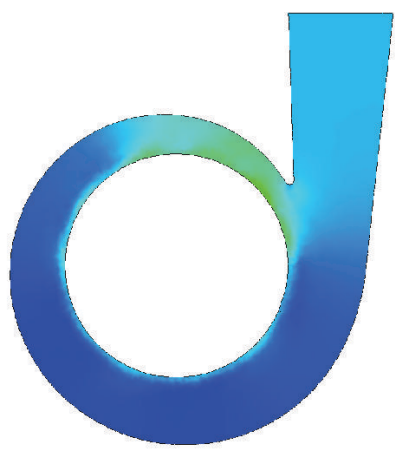

(c) $\mathrm{R} 1 \mathrm{AS}+\mathrm{V} 1$
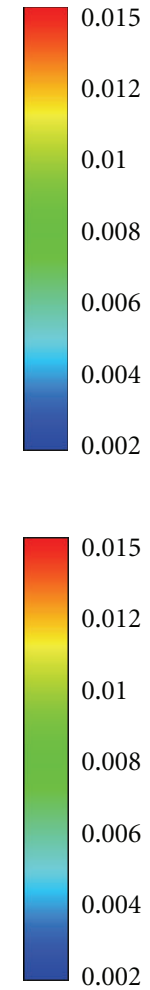

0.002

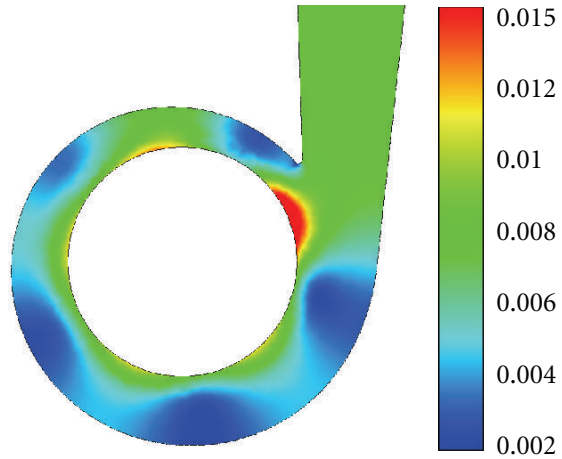

(b) $\mathrm{R} 1 \mathrm{~A}+\mathrm{V} 2$

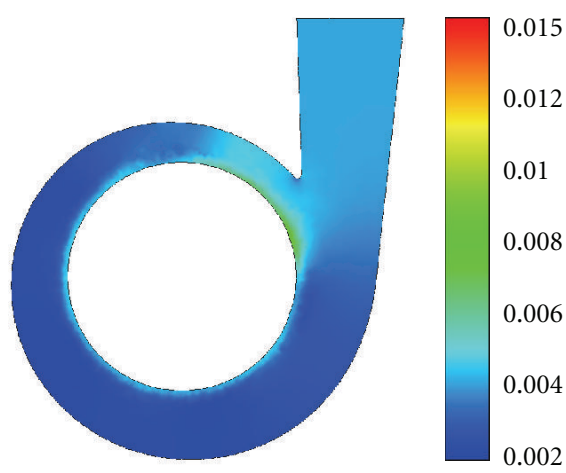

(d) R1AS+V2

Figure 12: Pressure fluctuation $\left(C_{\mathrm{pf}}\right)$ in the volute, at midspan of impeller outlet width.

own nominal flow rate. So, for the design point $\mathrm{R} 1 \mathrm{~A}+\mathrm{V} 1$ has the best performance concerning hydraulic efficiency, being $1 \%$ to $2 \%$ over the others. In this case, the differences are generated by the presence of shorten intermediates blades causing a decrease in hydraulic efficiency due mainly to the increase of hydraulic losses in the impeller blades channels and also by the distance between the tongue and the blades. The position of splitters in the middle of each bladed channel at the impeller outlet avoids blockage in the suction side of blade zone which will represent a velocity increase generating a static pressure drop. The comparison between $\mathrm{R} 1 \mathrm{~A}+\mathrm{V} 1$ and $\mathrm{R} 1 \mathrm{~A}+\mathrm{V} 2$ suggests that the incremented radial gap does not improve in any way the pump performance at the design flow rate and probably may not cause a substantial reduction in pressure fluctuations. Between the configuration R1AS+V1 (radial gap $=22 \%$ of impeller radius) and $\mathrm{R} 1 \mathrm{AS}+\mathrm{V} 2$ (radial gap $=25 \%$ of impeller radius), there is a gain in efficiency for the first one specially at high flow rates.

4.2. Instantaneous Static Pressure. The effects of geometrical changes in the morphology of the internal flow can be seen in Figure 7; it shows in detail the instantaneous static pressure contours for the four configurations when one main blade is near the volute tongue. The maximum pressure value is obtained at the outlet duct. It is caused by the energy conversion in the volute which has transformed the dynamic pressure into static pressure. The pump configurations with splitter blades (R1AS+V1 and R1AS+V2) present a more homogeneous circumferential pressure distribution except near the volute tongue which generates a zone of largest pressure gradient contributing to an unequal flow distribution in the blades.

4.3. Pressure Pulsations. In this section an analysis about the static pressure fields is carried out. The convergence of the unsteady calculations took approximately six impeller revolutions to achieve a periodic unsteady solution specially of the static pressure signals and ten revolutions to get enough data to have a good frequency resolution concerning the frequency analysis to be held later. In this way, the evolution in time of static pressure was recorded by means of virtual sensors placed, every $10^{\circ}$ at three different axial planes, one near the hub, another close to the shroud, and the last in the middle plane around the volute casing specially at the interface of the impellervolute (at diameter of 1.06 of $d_{2}$ ). The pulsating pressure at the inlet and the outlet pipe was captured by a set of 3 sensors installed at $600 \mathrm{~mm}$ from the inlet flange and outlet flange, respectively, and then averaged to present the fluctuating signal at each position. Figures 8 and 9 show the location of measurement points for two pump configurations. In order to compare the results between the four configurations, the position of the sensors was the same specially at inlet and at discharge pipe. The pressure signals were then analyzed and processed using the Fast Fourier Transform (FFT) with a Hanning Window providing a good resolution frequency. 


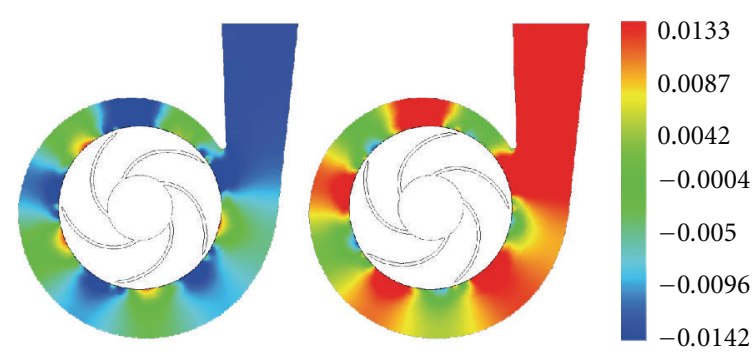

(a) $\mathrm{R} 1 \mathrm{~A}+\mathrm{V} 1$
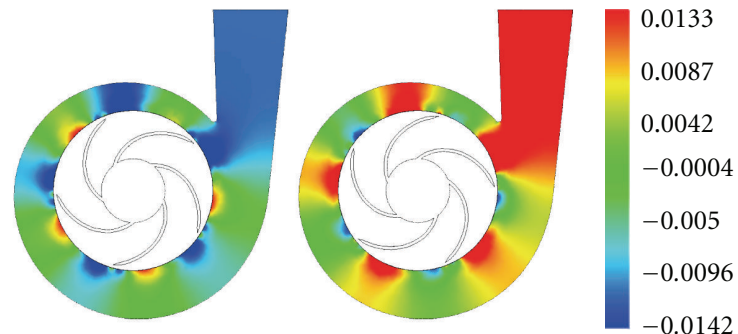

(b) $\mathrm{R} 1 \mathrm{~A}+\mathrm{V} 2$
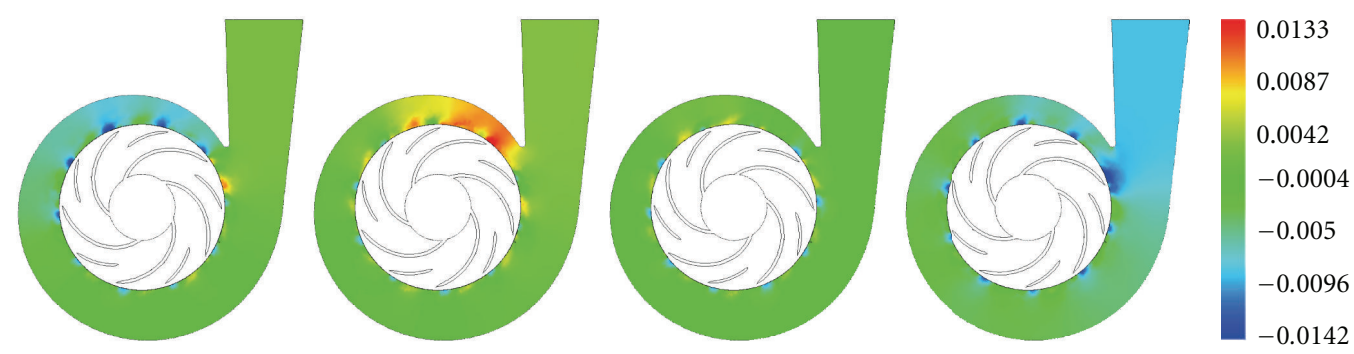

(c) R1AS+V1
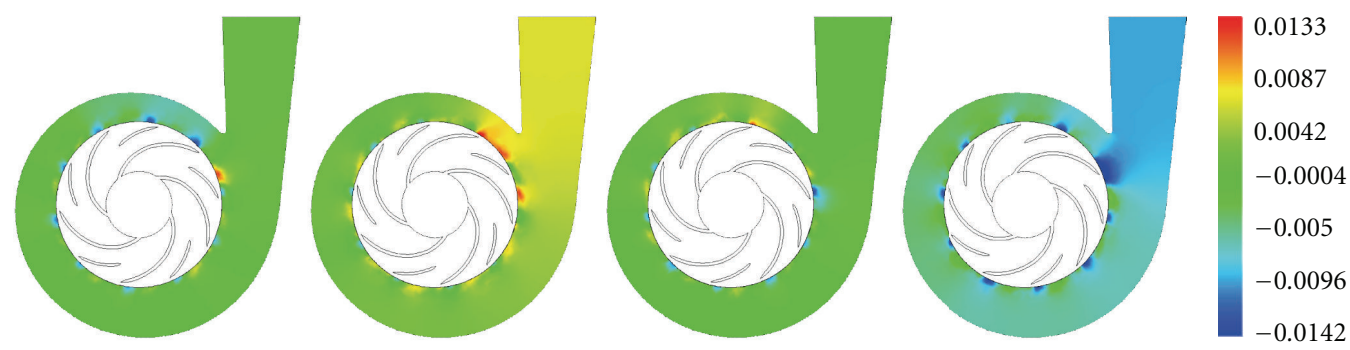

(d) R1AS+V2

FIGURE 13: Contours of normalized pressure fluctuation with respect to total head in the volute in relation of blades position.

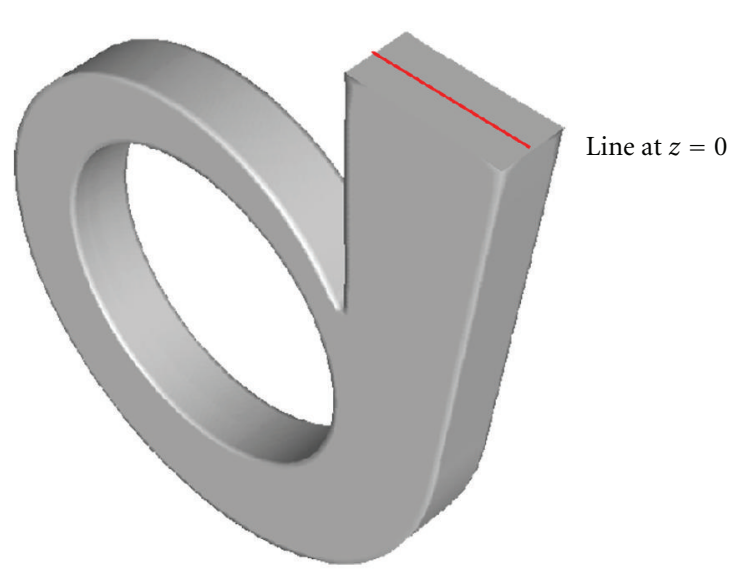

FIGURE 14: Line used as reference to place virtual sensors at volute discharge.

The numerical values extracted from the sensors inside the pump, representing the pressure signals generated by the interactions between the blades and the volute, are shown in Figure 10. The nonsymmetrical shape of the inner volute spiral as well as the divergent shape at pump discharge nozzle generate the appearance of a peak of strong amplitude near the tongue. The circumferential distribution of static pressure (normalized with $0.5 \rho U_{2}^{2}$ ) near impeller exit at the three axial planes at $t=0.5$ shows that there is no significant difference when the measurements are taken close to the wall or at the middle plane of the impeller outlet certifying that the values recorded at a distance of $Z=11.5$ or $Z=-11.5$ from the middle plane can be considered as representative values of the fluctuating signal at the impeller volute interface. For the configuration R1A+V1 and $\mathrm{R} 1 \mathrm{~A}+\mathrm{V} 2$, a sinusoidal behavior is observed, caused by the presence of the five blades, and also it can be noted the influence of radial gap (this modification has altered the volute tongue position) moving the highest peak to almost volute tongue position V2 at $\theta=40^{\circ}$. Concerning the pressure drop near the tongue, it can be observed that $\mathrm{R} 1 \mathrm{~A}+\mathrm{V} 1$ has a slope higher than $\mathrm{R} 1 \mathrm{~A}+\mathrm{V} 2$ which may have a negative effect on pressure pulsations. In relation to the geometries with intermediate vanes $(\mathrm{R} 1 \mathrm{AS}+\mathrm{V} 1$ and $\mathrm{R} 1 \mathrm{AS}+\mathrm{V} 2)$, the distribution is more uniform from $\theta=80^{\circ}$; and it keeps almost constant just before the tongue. Here, 


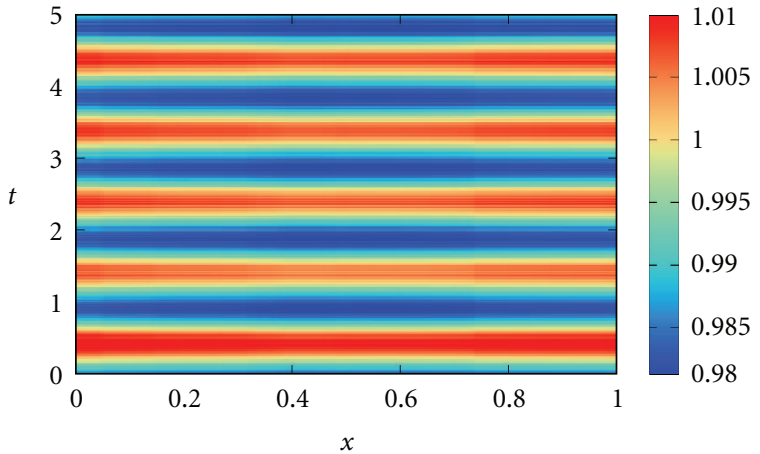

(a) $\mathrm{R} 1 \mathrm{~A}+\mathrm{V} 1$

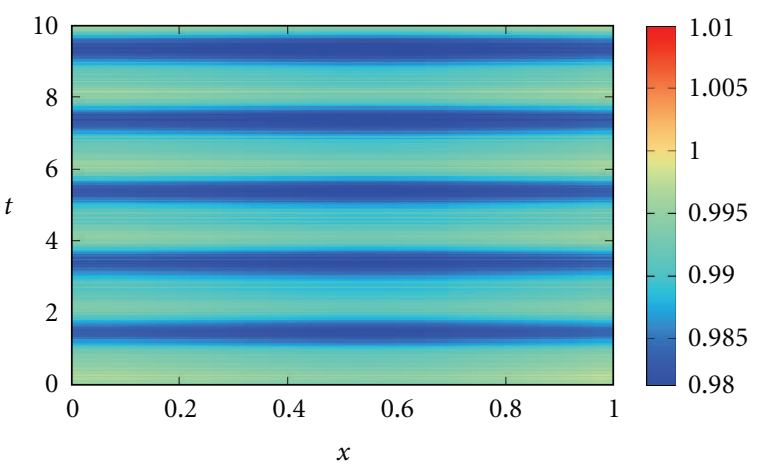

(c) $\mathrm{R} 1 \mathrm{AS}+\mathrm{V} 1$

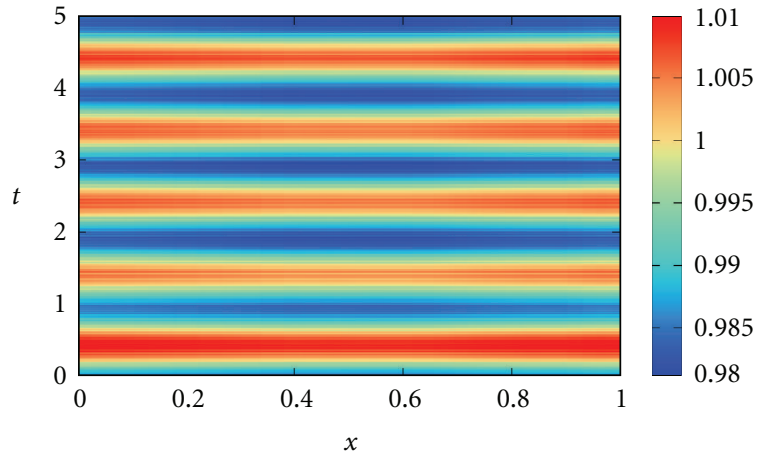

(b) $\mathrm{R} 1 \mathrm{~A}+\mathrm{V} 2$

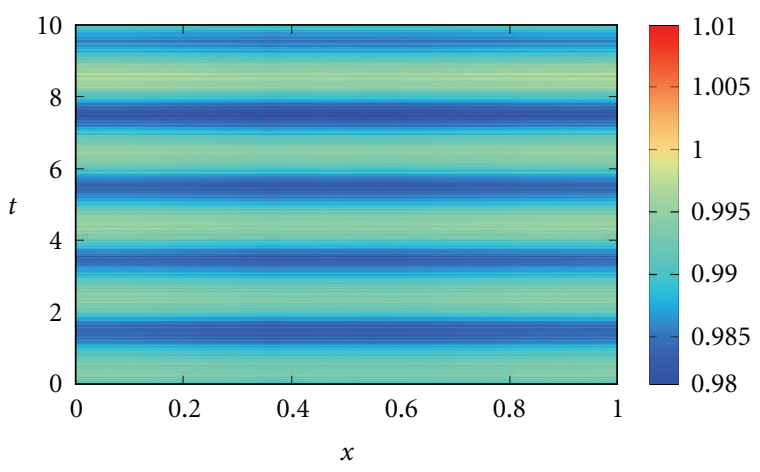

(d) R1AS+V2

FIGURE 15: Contours of normalized static pressure with respect to total head at volute discharge during one impeller revolution.

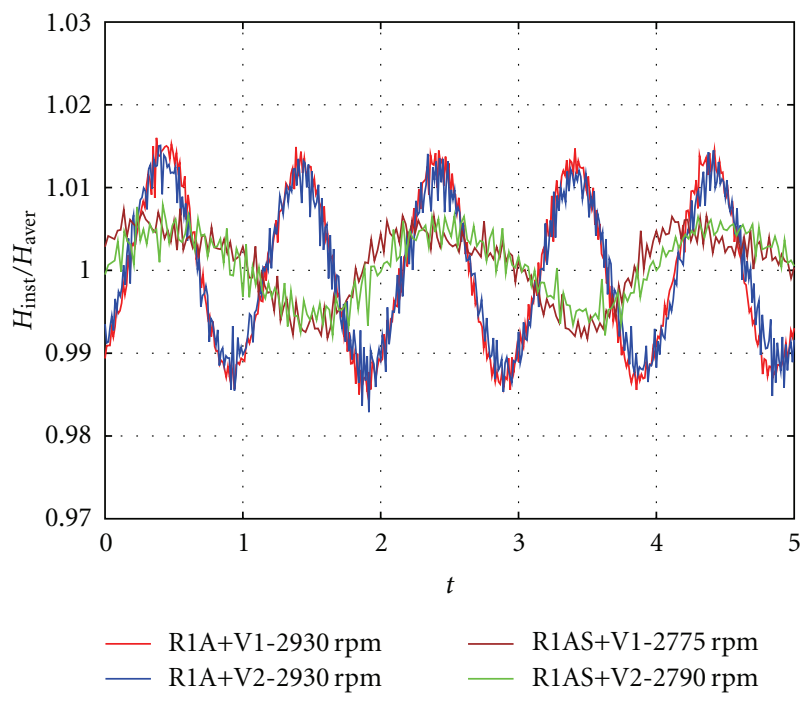

FIGURE 16: Comparison of ratio between the instantaneous total head and the average total head for the four pump configurations.

the same phenomena is observed concerning the slope, being more high for the configuration with the volute V1.

The time-space behavior of static pressure just after the impeller exit is presented in Figure 11, the static pressure values were collected using a circumferential line positioned at 1.06 of $d_{2}$ and at the middle plane of the impeller.
The pressure is nondimensionalized with respect to $0.5 \rho U_{2}{ }^{2}$ and then represented as a contour plot. The $x$-coordinate presents $\theta$ that is the angular position around the impeller, and the $y$-coordinate corresponds to the normalized time $t$. The numerical results were recorded from the instant that the main blade is in front of the volute tongue $(t=0)$ until the next one occupies the same position ( $t=1$ for R1A and $t=2$ for R1AS). Thus, each plot details a single blade passage (main blades) where the tongue position is indicated by a dashed line which depends on the volute tongue position being $\theta=35^{\circ}$ for $\mathrm{V} 1$ and $\theta=40^{\circ}$ for $\mathrm{V} 2$. So, it can be seen that the time history of the nondimensional magnitude of the static pressure at any any angular will have a periodic behavior related to the blade passage, noticing that the largest amplitudes are localized near the tongue more precisely behind it as a consequence of volute shape. On the other hand, the configuration R1AS+V2 presents the lowest fluctuating pressure compared to the others, showing a very slight variation at any $\theta$.

The distribution of pressure fluctuations at $Z=0$ in the volute for the tested pumps is plotted in Figure 12. One impeller revolution is used to get transient statics of the numerical results and to record the static pressure on the volute computational domain. To determine the magnitude of the fluctuating pressure, a pressure fluctuation coefficient $\left(C_{\mathrm{pf}}\right)$ is used, which is calculated by the RMS (Root Mean Square) of unsteady pressures normalized by the dynamic pressure $0.5 \rho U_{2}{ }^{2}$. The comparison between 


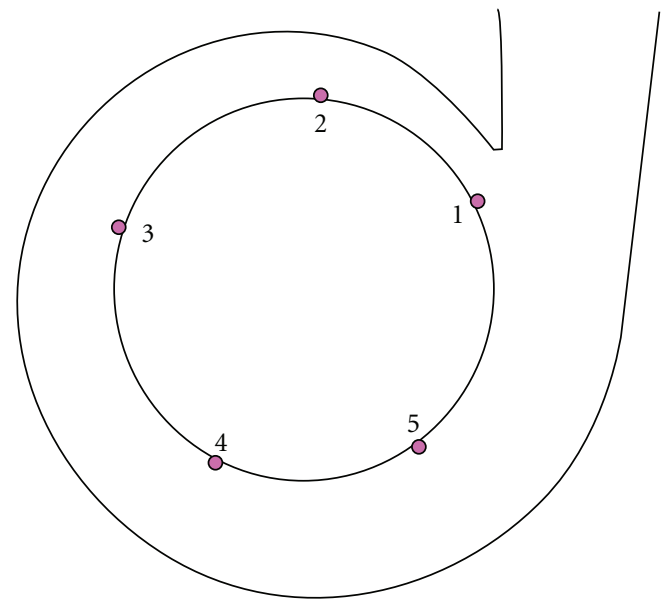

(a) Volute V1

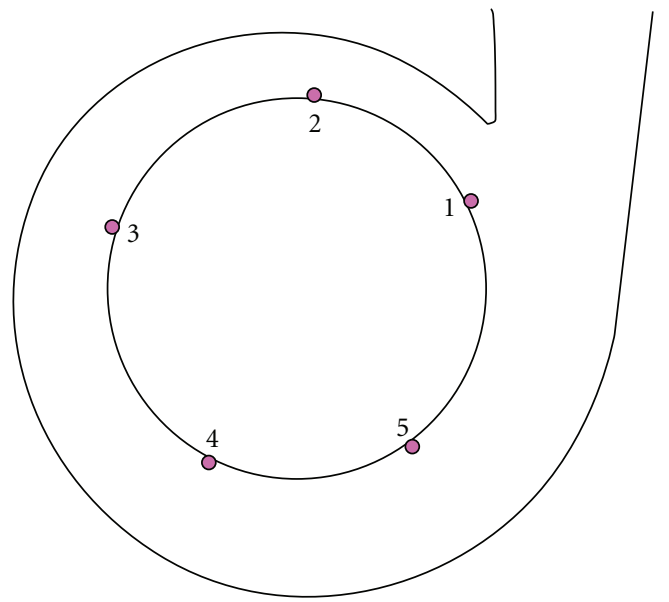

(b) Volute V2

FIGURE 17: Angular positions of virtual sensors at interface impeller volute.

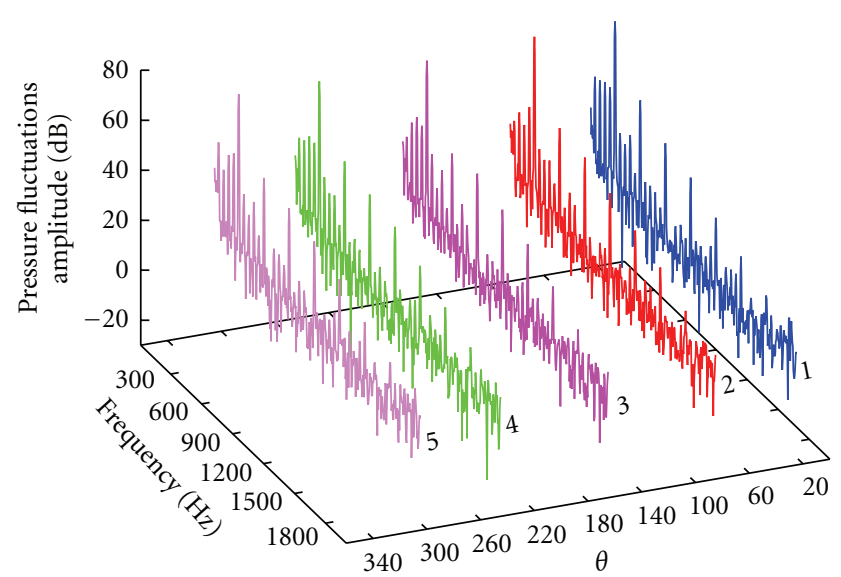

(a) $\mathrm{R} 1 \mathrm{~A}+\mathrm{V} 1$
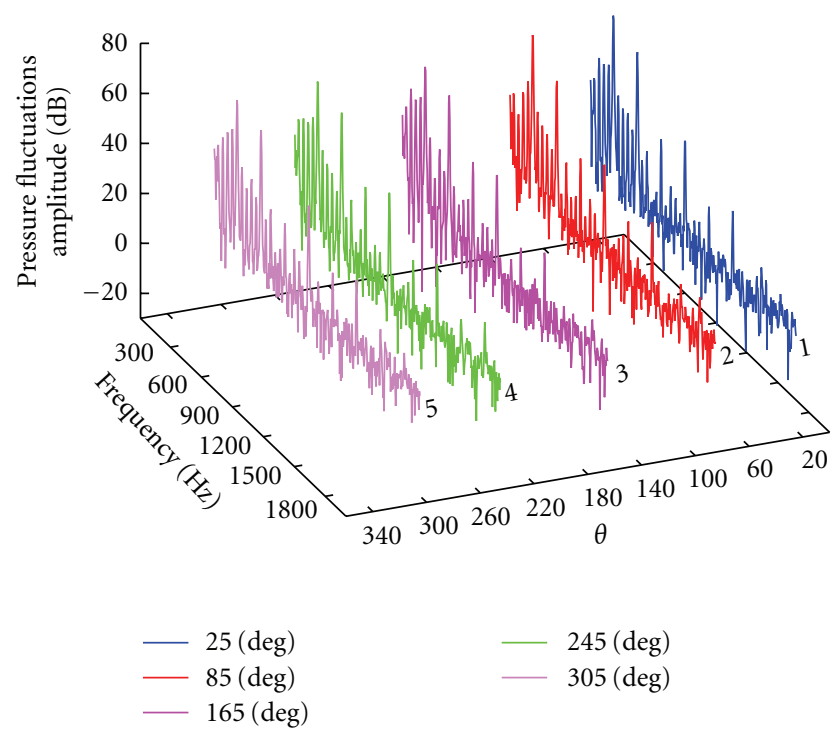

(c) R1AS+V1

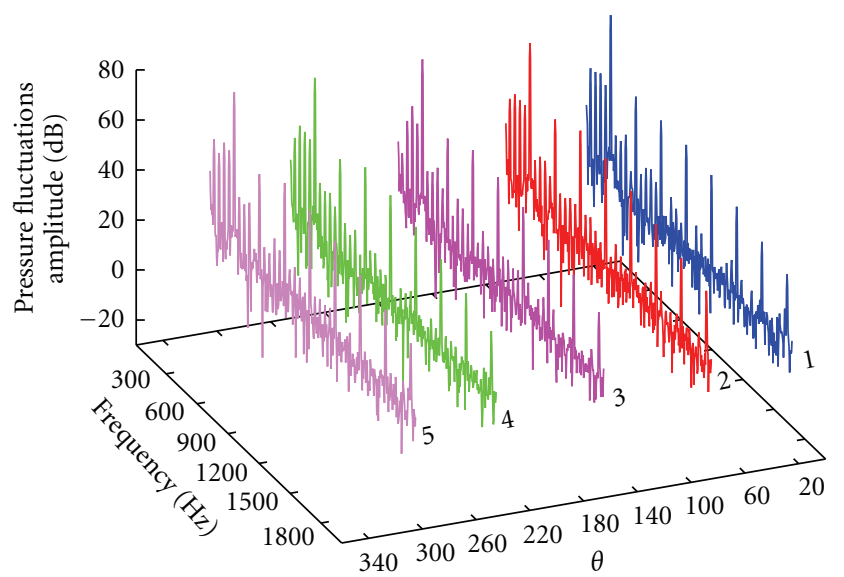

(b) $\mathrm{R} 1 \mathrm{~A}+\mathrm{V} 2$

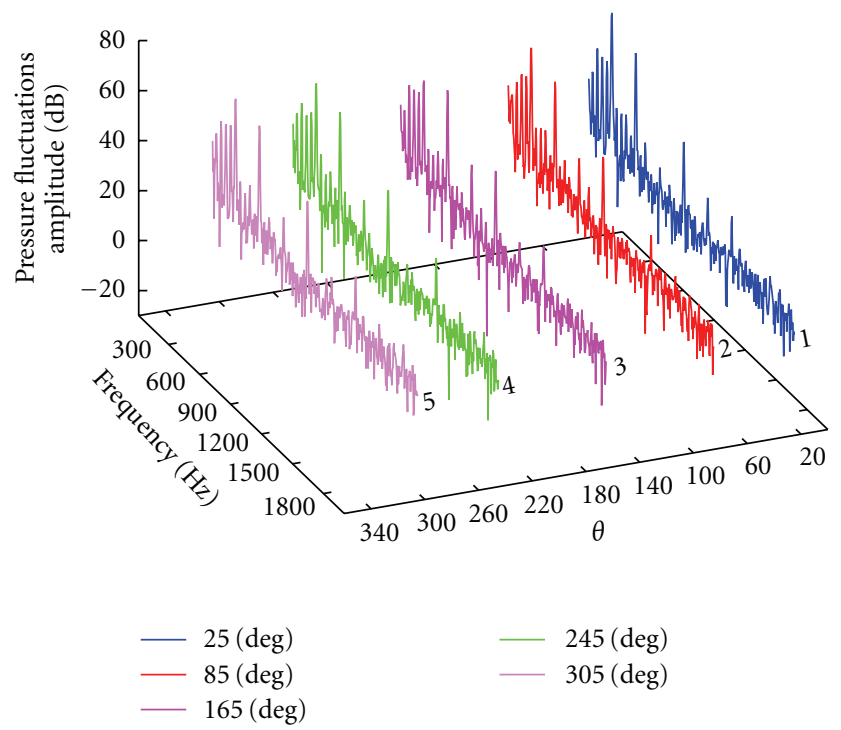

(d) R1AS+V2

Figure 18: Pressure pulsations spectra for the pump configurations at various angular positions around the impeller exit $\left(P_{\text {ref }}=1 \mathrm{~Pa}\right)$. 


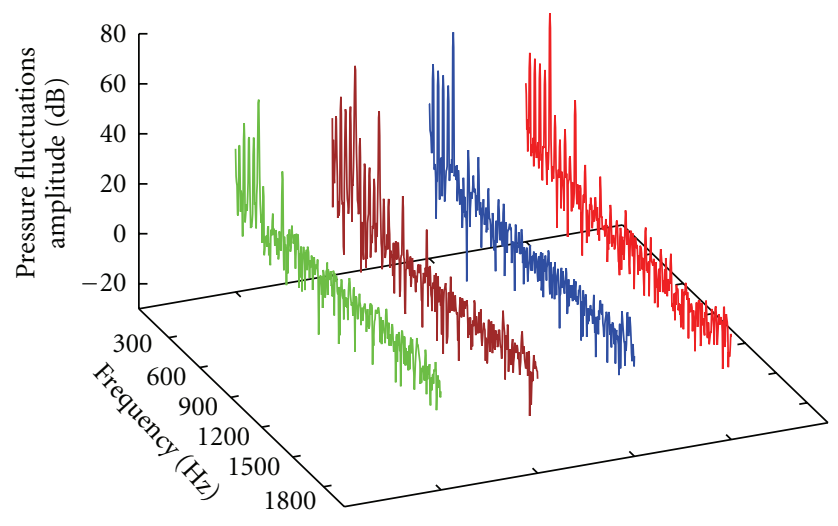

- R1A+V1-2930 rpm

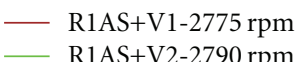

FIGURE 19: Comparison of static pressure pulsations levels in the frequency domain between the four pump configurations $\left(P_{\text {ref }}=\right.$ $1 \mathrm{~Pa})$ in the outlet pipe.
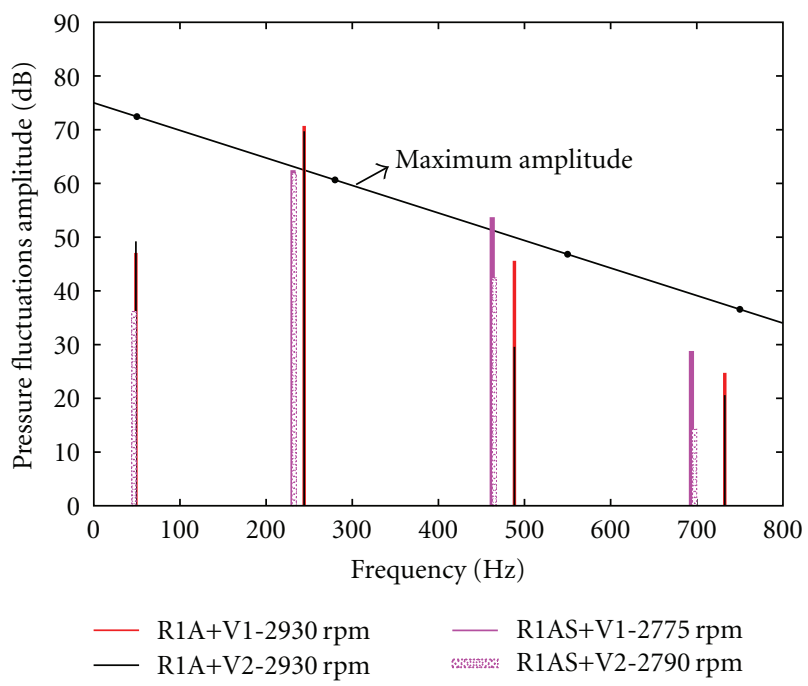

Figure 20: Pressure spectrum of static pressure pulsations at discharge duct for the four pump configurations $\left(P_{\text {ref }}=1 \mathrm{~Pa}\right)$.

the four configurations reveals clearly a reduction of fluctuations as we introduce a geometric modification in the reference pump. The biggest pressure fluctuation is observed in $\mathrm{R} 1 \mathrm{~A}+\mathrm{V} 1$ reaching up to 0.015 ; it is localized in two regions near the volute tongue as well as in $\mathrm{R} 1 \mathrm{~A}+\mathrm{V} 2$ in which the increment of the radial gap has reduced the fluctuations in regions far from the volute tongue but enlarging the strong fluctuation zone near it. In the case of R1AS+V2, the pressure fluctuation is lower than the others, having a more homogeneous distribution around the volute. It is also found that there are some regions that present smaller pressure fluctuations in relation to the volute discharge.

These pressure fluctuations can be examined with more details by comparing them in relation to the blades position. For this purpose Figure 13 is plotted to represent

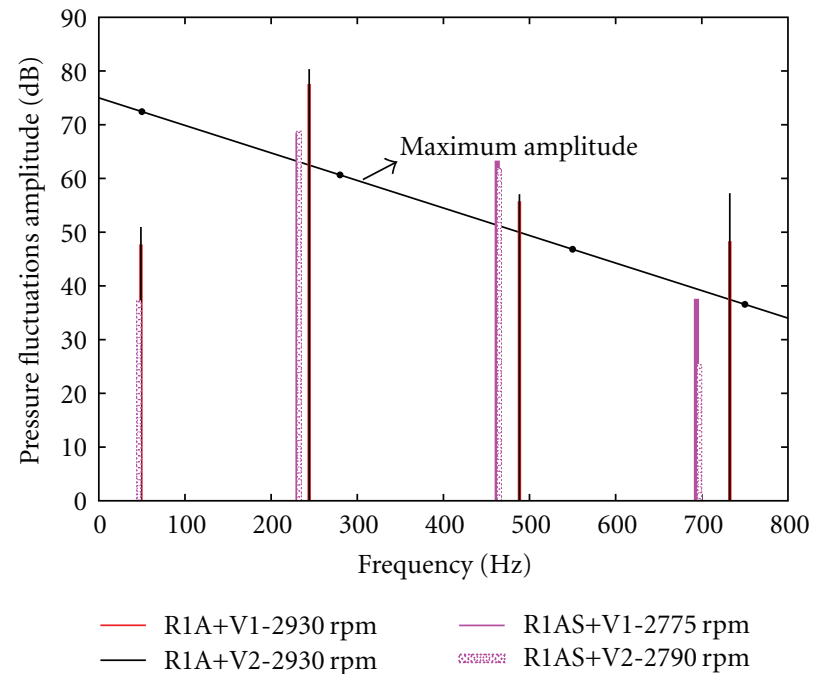

FIGURE 21: Pressure spectrum of static pressure at interface impeller volute $\theta=25^{\circ}$ for the four pump configurations $\left(P_{\text {ref }}=1 \mathrm{~Pa}\right)$.

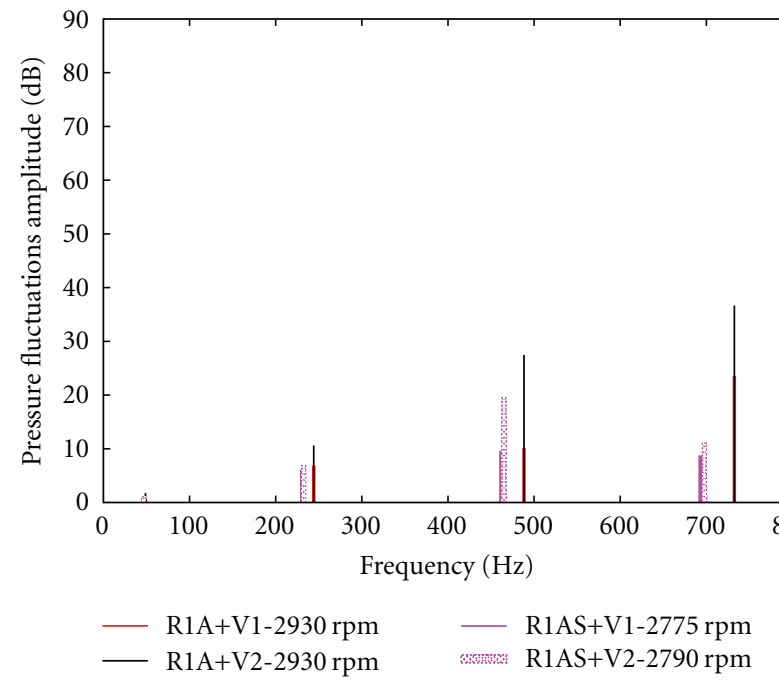

FIGURE 22: Reduction of static pressure levels generated by the volute for the four pump configurations $\left(P_{\text {ref }}=1 \mathrm{~Pa}\right)$.

the effects of the impeller blade position on unsteady pressures. In the four configurations, the approach of mean blade leading edge to the tongue and the intermediate position of a splitter-blade channel respect to the tongue causes low fluctuations in the volute. The highest ones are observed when the tongue is between the main blades and when it is between a blade-splitter channel. In this way, we can verify the influence of adding intermediate blades on pressure fluctuations reduction.

To better understand in a better way the attenuation and the propagation of pressure fluctuations inside the pump, a straight horizontal line formed by various sensors was created at volute discharge (see detail in Figure 14 red line). The data collected during an impeller revolution is illustrated in 
TABLE 3: Numerical values of pressure fluctuations amplitudes in $\mathrm{dB}$ for the investigated configurations $\left(P_{\text {ref }}=1 P a\right)$.

\begin{tabular}{lccc}
\hline Configuration & $F / F_{\mathrm{BP}}$ & $\theta=25^{\circ}(\mathrm{dB})$ & Outlet duct $(\mathrm{dB})$ \\
\hline \multirow{2}{*}{ R1A+V1 } & 0.2 & 47.72 & 47.07 \\
& 1 & 77.58 & 70.71 \\
& 2 & 55.75 & 45.60 \\
& 3 & 48.32 & 24.75 \\
\hline R1A+V2 & 0.2 & 50.97 & 49.23 \\
& 1 & 80.35 & 69.72 \\
& 2 & 57.07 & 29.58 \\
R1AS+V1 & 3 & 57.26 & 20.60 \\
& 0.1 & 35.11 & 34.99 \\
& 1 & 68.58 & 62.47 \\
& 1.5 & 63.33 & 53.71 \\
R1AS+V2 & 0.1 & 37.60 & 28.82 \\
\hline & 0.5 & 68.79 & 36.16 \\
& 1 & 61.89 & 61.96 \\
& 1.5 & 25.32 & 42.40 \\
& & & 14.25 \\
\hline
\end{tabular}

Figure 15 showing the contours of instantaneous static pressure nondimensionalized with respect to the average total head of each configuration. The coordinate in $x$ direction represents the normalized distance along the line, and the coordinate in $y$ corresponds to the blade passage normalized by time. The comparison between the four configurations shows a spatial uniform propagation following the line, and a time periodic pattern for all geometries can be seen during an impeller revolution. The transition between the contours colors in R1AS+V2 and R1AS+V1 is much smaller than $\mathrm{R} 1 \mathrm{~A}+\mathrm{V} 1$ and $\mathrm{R} 1 \mathrm{~A}+\mathrm{V} 2$ giving the idea that they will generate low-amplitude pulsating signals. This observation will be confirmed later by a frequency analysis. In the case of an impeller with splitters, five peaks are still perceptible corresponding to a certain way to blades passage, while it seems that the other peaks were merged or attenuated in the volute. For the impeller geometry without splitters, the five peaks appear more clearly, when the blade is approaching the tongue. Additionally, Figure 16 reveals the fluctuating signals recorded by the sensors at the outlet channel; it illustrates a part of the complete signal nondimensionalized with respect to the averaged head. The comparisons show that the splitter blades decrease the pressure amplitudes levels. The influence of the radial gap between the configurations using the same impeller R1A or R1AS is not visible. An FFT analysis will give more details about this aspect.

\section{Numerical Frequency Analysis}

Computational studies were performed for the four pumps of which the first one is the hydraulic which will be optimized, and the others are the improved versions. The differences lie primarily in the radial gap distance and also the blades number at the impeller periphery. Thus, the fluctuating pressures recorded during the operation of the pump are composed essentially by the signals at the interface of the impeller volute, at the inlet and at the outlet duct. In order to have a better understanding and to compare in the frequency domain the influence of the geometrical changes at the impeller periphery, five additional angular positions (Figure 17) were chosen near the shroud at $Z=$ $11.5 \mathrm{~mm}$. Figure 18 shows the pressure pulsations spectra at the positions detailed above, presenting several peaks dominated mainly by tones at the blade passage frequency $F_{\mathrm{BP}}$ and their harmonics where the amplitude levels are shown as Pressure Fluctuations Amplitudes in $\mathrm{dB}$ using a Reference Pressure $P_{\text {ref }}$ equal to $1 \mathrm{~Pa}$. It should be noted that the appearance of these peaks is mainly due to the interactions between the periodic flow discharging from the impeller and the volute. The differences encountered between the static pressure pulsations levels generated by each configuration are mainly due to geometrical changes that have decreased the internal interactions specially near the volute tongue and they approach the discharge channel; as well as this result shows the importance of providing specific impeller geometry to achieve a desired pressure fluctuation spectra. Concerning the fluctuations at the outlet duct, Figure 19 illustrates the reduction of amplitudes with values lower than those found within the pump. Table 3 resumes the processed values by means of FFT and the Hanning Window for the angular position of $\theta=25^{\circ}$ (behind the tongue) and for the pressure signal at outlet duct, where $F_{\mathrm{BP}}$ is referring to the fundamental peak generated by the blades $z\left(=z_{m}\right.$ for R1A, $=z_{m}+z_{s}$ for R1AS). According to the final results, R1AS+V2 is the best pump configuration to decrease pressure fluctuations at the same operating point of the reference geometry, generating low pulsations than the others at the blade passage frequency as well as at the harmonic frequencies, and so validating the fact that adding intermediate blades and incrementing the distance blades tongue are necessary to decrease pressure amplitudes levels specially at the outlet canal; in relation to the radial gap influence (volute $\mathrm{V} 1$ and volute $\mathrm{V} 2$ ) on the pressure fluctuations at discharge duct. Between $\mathrm{R} 1 \mathrm{~A}+\mathrm{V} 1$ and $\mathrm{R} 1 \mathrm{~A}+\mathrm{V} 2$ is found that the volute V2 has a more important influence on the reduction of the harmonic peaks than the fundamental one, the same behavior is observed when R1AS+V1 is compared to R1AS+V2. Noticing that incrementing radial gap is more effective when the impellers contain splitters. Concerning the effect of splitters, they act on the attenuations specially the fundamental peak of R1A+V1 as we can seen in Figure 19. There is a reduction of approximately $9 \mathrm{~dB}$ considering any configuration containing the impeller R1AS compared to the reference pump. The only problem caused by the addition of these intermediates blades is that they do emerge the harmonics when no modification is made to radial gap. Through the numerical results and the processed signals at the interface impeller volute for an angular position of $\theta=25^{\circ}$, the effect of incrementing the radial gap causes a slight increase on pressure amplitudes between $\mathrm{R} 1 \mathrm{~A}+\mathrm{V} 1$ and $\mathrm{R} 1 \mathrm{~A}+\mathrm{V} 2$ at $F_{\mathrm{BP}}$. In relation to R1AS+V1 and R1AS+V2, the influence of radial gap is specially noticed in the harmonics. The above differences can be seen more clearly in Figures 20 to 22 that present the pressure spectra comparison between 
the four configurations at $\theta=25^{\circ}$, at outlet canal and the contribution of the volute geometry to attenuate the static pressure levels. It should be pointed out that even though $\mathrm{R} 1 \mathrm{AS}+\mathrm{V} 1$ attenuates the fundamental peak of $\mathrm{R} 1 \mathrm{~A}+\mathrm{V} 1$, it does rise the first and second harmonic. A solution to avoid this is to increment the radial gap a little more. Thus a new volute V2 is used to decrease the harmonics in order to obtain smaller amplitudes than the original configuration. Hence, to choose the best hydraulic which exhibits low pressure fluctuations characteristics, an industrial specification is considered as reference that corresponds to the characteristics of an industrial pump called $\mathrm{V} 0$, the pressure fluctuations amplitudes were obtained after experimental tests. In the context of this study, the search for a new pump based on geometrical optimizations in terms of pressure fluctuations leads us to make geometric changes to R1A+V1 in order to respect the industrial specification (maximum amplitude). The four configurations were compared in relation to the maximum amplitude (see Figures 20 and 21), showing specially at discharge duct; that R1AS+V2 is the hydraulic configuration with lowest pressure amplitudes at blade passage frequency of the reference pump. Thus, there are many factors to consider which have a direct influence on the distribution of peaks in the pressure spectrum. The fact of adding five splitters to the original impeller attenuates the fundamental peak of R1A, but does rise the first harmonic. So, to decrease the pressure fluctuations amplitudes of this one, increasing the radial gap is necessary and has also a direct influence on the fundamental peak generated by the impeller R1AS. Regarding the pressure fluctuations reduction caused by the volute (Figure 22), it is very difficult to establish a relation considering the results in order to determine how much it will decrease the amplitude levels.

\section{Conclusions and Perspectives}

Four pump configurations were tested using numerical simulations in order to optimize the initial hydraulic concerning pressure fluctuations by means of geometrical modifications on the impeller and volute casing. For this purpose, three different geometries were built from the reference pump and were analyzed at the same operating condition of the baseline. Virtual sensors collected the fluctuating pressure signal in various positions and provided detail information regarding the pressure fluctuation evolution in the volute and at inlet and at discharge pipe. Thus, the following conclusions can be deduced from the numerical study.

(i) The largest pressure fluctuation occurs at the impeller exit specially near the volute tongue.

(ii) Comparisons among the four pump configurations show the importance of adding intermediary blades in the impeller, giving better results and being more effective for decreasing pulsations levels and also supplemented with an appropriate increase of radial gap. (iii) The election of suitable boundary conditions is essential to predict effectively the pressure fluctuations specially at the pump discharge. The choice of outflow boundary condition at the outlet of the domain is motivated by the fact that the velocity and pressure are not known and imposing a pressure outlet condition will have an numerical impact on the pressure fluctuations.

(iv) The most remarkable result is that the optimized geometry R1AS+V2 has an advantage in the amplitude of pressure pulsation reducing considerably the amplitudes in the outlet pipe by about $9 \mathrm{~dB}$ at $F / F_{\mathrm{BP}}=1$ of $\mathrm{R} 1 \mathrm{~A}+\mathrm{V} 1$. Considering the ratio between the instantaneous total head and the average total head for the pumps configurations, it can be concluded that the pressure fluctuations represent approximately $0.5 \%$ of the average head in R1AS+V2, while for $\mathrm{R} 1 \mathrm{~A}+\mathrm{V} 1$ are about $1.5 \%$.

Finally, concerning the interest of decrease pressure pulsation into the pump and specially in the outlet pipe at the main frequency of reference pump, numerical studies will be complemented with experimental tests to confirm the numerical results.

\section{Nomenclature}

\begin{tabular}{|c|c|}
\hline$b_{2}:$ & Impeller width at outlet ( $\mathrm{mm})$ \\
\hline$b_{3}:$ & Volute width at base circle ( $\mathrm{mm})$ \\
\hline$C_{\mathrm{pf}}:$ & Pressure fluctuation coefficient \\
\hline$d_{1}:$ & Impeller diameter at inlet (mm) \\
\hline$d_{2}:$ & Impeller diameter at outlet ( $\mathrm{mm})$ \\
\hline$d_{3}:$ & Volute base circle diameter (mm) \\
\hline$F:$ & Frequency $(\mathrm{Hz})$ \\
\hline$F_{\mathrm{BP}}:$ & $\begin{array}{l}\text { Blade passage frequency }(\mathrm{Hz}), \\
F_{\mathrm{BP}}=z F_{R}\end{array}$ \\
\hline$F_{R}:$ & Rotational frequency $(\mathrm{Hz}), F_{R}=N / 2 \pi$ \\
\hline$H, H_{N}:$ & $\begin{array}{l}\text { Total head and total head at nominal } \\
\text { point }[\mathrm{m}]\end{array}$ \\
\hline$H_{\text {inst }}, H_{\text {aver }}$ : & $\begin{array}{l}\text { Instantaneous head and averaged head } \\
{[\mathrm{m}]}\end{array}$ \\
\hline$N:$ & Angular velocity (rad/s) \\
\hline$P_{s}:$ & Static pressure $(\mathrm{Pa})$ \\
\hline$P_{\text {ref: }}$ & Reference pressure $(\mathrm{Pa})$ \\
\hline$Q, Q_{N}:$ & $\begin{array}{l}\text { Flow rate and flow rate at nominal } \\
\text { point }\left(\mathrm{m}^{3} / \mathrm{h}\right)\end{array}$ \\
\hline$t:$ & $t_{\text {inst }} / t_{\mathrm{BP}}$, normalized time \\
\hline$t_{\mathrm{BP}}:$ & $1 / F_{\mathrm{BP}}$, period of blade passage $(\mathrm{s})$ \\
\hline$t_{\text {inst }}:$ & Instantaneous time (s) \\
\hline$U_{2}:$ & $\begin{array}{l}\text { Circumferential velocity at impeller } \\
\text { outlet }(\mathrm{m} / \mathrm{s})\end{array}$ \\
\hline$x:$ & Normalized distance \\
\hline$z:$ & $z_{m}+z_{s}$, total blades number \\
\hline$z_{m}:$ & Main blades number \\
\hline$z_{s}:$ & Splitter blades number \\
\hline$\eta:$ & Hydraulic efficiency \\
\hline$\rho:$ & Density $\left(\mathrm{kg} / \mathrm{m}^{3}\right)$ \\
\hline$\theta:$ & $\begin{array}{l}\text { Circumferential position around the } \\
\text { impeller (deg). }\end{array}$ \\
\hline
\end{tabular}




\section{Subscript}

1: Impeller inlet

2: Impeller outlet

3: Volute inlet

aver: Averaged

BP: Blade passage

inst: Instantaneous

$m: \quad$ Main

$N$ : Nominal

pf: Pressure fluctuation

$R$ : Rotational

ref: Reference

$s: \quad$ Splitter.

\section{References}

[1] J. S. Choi, D. K. McLaughlin, and D. E. Thompson, "Experiments on the unsteady flow field and noise generation in a centrifugal pump impeller," Journal of Sound and Vibration, vol. 263, no. 3, pp. 493-514, 2003.

[2] K. Majidi, "Numerical study of unsteady flow in a centrifugal pump," Journal of Turbomachinery, Transactions of the ASME, vol. 127, no. 2, pp. 363-371, 2005.

[3] R. Spence and J. Amaral-Teixeira, "Investigation into pressure pulsations in a centrifugal pump using numerical methods supported by industrial tests," Computers and Fluids, pp. 1-15, 2007.

[4] M. Asuaje, F. Bakir, S. Kouidri, F. Kenyery, and R. Rey, "Numerical modelization of the flow in centrifugal pump: volute influence in velocity and pressure fields," International Journal of Rotating Machinery, vol. 3, pp. 244-255, 2005.

[5] G. Kergourlay, M. Younsi, F. Bakir, and R. Rey, "Influence of splitter blades on the flow field of a centrifugal pump: test-analysis comparison," International Journal of Rotating Machinery, vol. 2007, Article ID 85024, 13 pages, 2007.

[6] H. Miyamoto, Y. Nakashima, and H. Ohba, "Effects of splitter blades on the flows and characteristics in centrifugal impellers," JSME International Journal, vol. 35, no. 2, pp. 238246, 1992.

[7] J. L. Parrondo-Gayo, J. González-Pérez, and J. FernándezFrancos, "The effect of the operating point on the pressure fluctuations at the blade passage frequency in the volute of a centrifugal pump," Journal of Fluids Engineering, Transactions of the ASME, vol. 124, no. 3, pp. 784-790, 2002.

[8] S. Chu, R. Dong, and J. Katz, "Relationship between unsteady flow, pressure fluctuations, and noise in a centrifugal pumppart a: effects of blade-tongue interactions," Journal of Fluids Engineering, Transactions of the ASME, vol. 117, pp. 24-29, 1995.

[9] S. Chu, R. Dong, and J. Katz, "Relationship between unsteady flow, pressure fluctuations, and noise in a centrifugal pumppart B: effects of blade-tongue interactions," Journal of Fluids Engineering, Transactions of the ASME, vol. 117, no. 1, pp. 30 35, 1995.

[10] R. Dong, S. Chu, and J. Katz, "Effect of modification to tongue and impeller geometry on unsteady flow, pressure fluctuations, and noise in a centrifugal pump," Journal of Turbomachinery, Transactions of the ASME, vol. 119, no. 3, pp. 506-515, 1997.

[11] J. González, J. Parrondo, C. Santolaria, and E. Blanco, "Steady and unsteady radial forces for a centrifugal pump with impeller to tongue gap variation," Journal of Fluids Engineering, Transactions of the ASME, vol. 128, no. 3, pp. 454462, 2006.

[12] R. Barrio, E. Blanco, J. Parrondo, J. González, and J. Fernández, "The effect of impeller cutback on the fluid-dynamic pulsations and load at the blade-passing frequency in a centrifugal pump," Journal of Fluids Engineering, Transactions of the ASME, vol. 130, no. 11, pp. 1111021-11110211, 2008.

[13] "Fluent 6.3.26," http://www.fluent.com.

[14] M. Solis, S. Khelladi, and F. Bakir, "A new-high precision solver to predict pressure fluctuations in centrifugal pumps," in Proceedings of the ASME-JSME-KSME Conference, p. 13, 2011. 

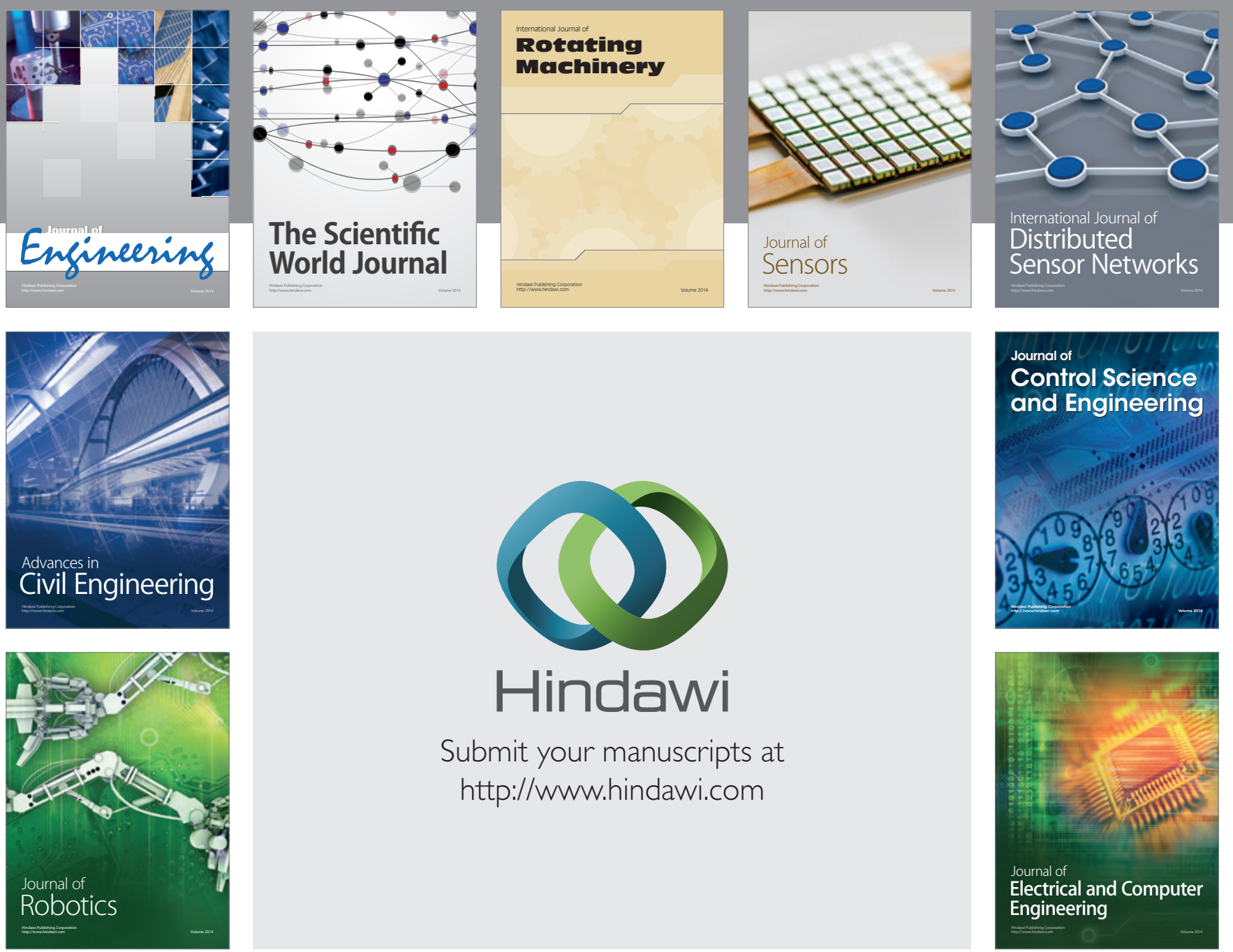

Submit your manuscripts at

http://www.hindawi.com
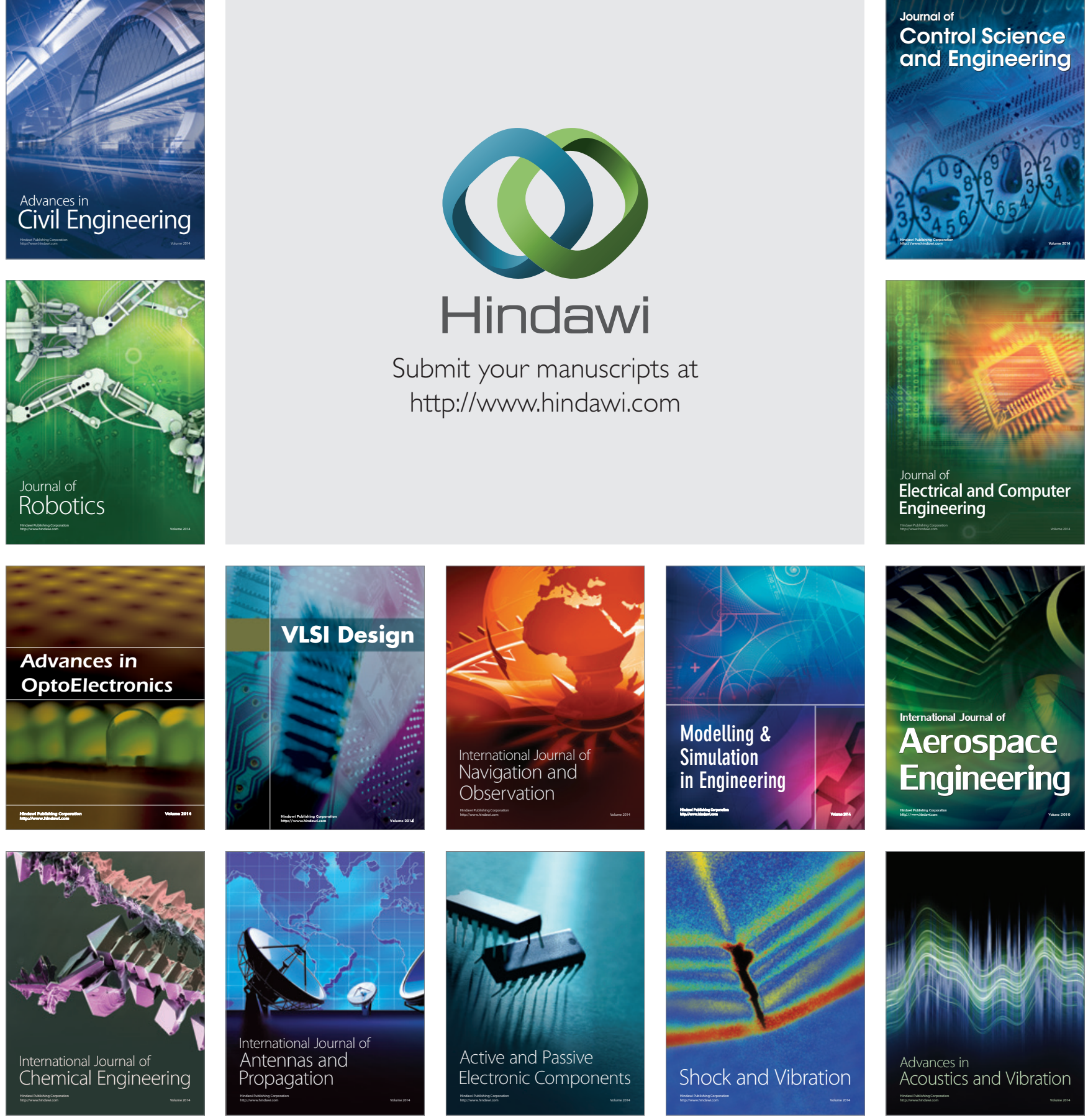